\title{
El Poder Judicial en discurso. Controversias en la presentación del Proyecto de Democratización de la Justicia en Argentina
}

Recibido: 14/07/2021

Aprobado: 18/09/2021
IRENE LIS GINDIN

CONICET-Centro de Investigaciones en Mediatizaciones

Universidad Nacional de Rosario (UNR)

irene.gindin@conicet.gov.ar

https://orcid.org/0000-0002-3080-0207

\begin{abstract}
RESUMEN
Ante el avance de la judicialización de la política —y la politización de la justicia—, este artículo se propuso recrear una escena controversial. Controversia, debate, litigio y polémica fueron categorías utilizadas en este texto para caracterizar el intercambio - tanto político como periodístico- producido en torno al Proyecto de Democratización de la Justicia presentado por la expresidenta de Argentina, Cristina Fernández de Kirchner, en el año 2013. Para ello, hemos analizado las noticias referidas al proyecto de tres diarios de circulación nacional (Clarín, La Nación y Página/12) y las pusimos en tensión con el discurso propiamente político. Lo importante en este punto no consiste tanto en la verificación de si lo estudiado se corresponde efectivamente con un proceso de lawfare o, a la inversa, con un intento de un determinado Gobierno de interceder - con fines político partidarios - en el correcto funcionamiento de la justicia sino, más bien, cómo esos procesos político-judiciales que estudiamos aparecen así tematizados en la escena pública argentina. Los materiales fueron analizados considerando, como hipótesis, que la discusión se estructura en torno a tres ejes: i) el momento en el que es planteada la reforma, ii) la discusión sobre su constitucionalidad, y iii) el debate sobre la división de poderes.
\end{abstract}

Palabras clave: Lawfare, poder judicial, kirchnerismo, república

\section{The Judiciary in discourse. Controversies in the presentation of the Project for the Democratization of Justice in Argentina}

\begin{abstract}
Faced with the advance of the judicialization of politics — and the politicization of justice- , this article set out to recreate a controversial scene. Controversy, debate, litigation and polemic were categories used in this text to characterize the exchange - both political and journalistic - around the Project for the Democratization of Justice presented by the former president of Argentina, Cristina Fernández de Kirchner, in 2013. To this end, we have analyzed the news of three national newspapers (Clarin, La Nación and Página/12) related to the project and match them up against the actual political discourse. The importance of this analysis lies not in verifying whether the study actually corresponds to a lawfare process or, conversely, to an attempt by a certain government to intercede for political party purposes - in the correct functioning of justice. Rather than in how those political-
\end{abstract}


judicial processes that we study appear thus thematized in the Argentine public scene. The materials were analyzed considering, as a hypothesis, that the discussion is structured around three axes: i) the moment in which the reform is proposed, ii) the discussion on its constitutionality, and c) the debate on the division of powers.

Keywords: Lawfare, judiciary, Kirchnerism, republic

¿Por qué el 'lawfare’ es un cuento?» ${ }^{1}$ «Judicialización de la política y polí-
tica judicial»,${ }^{2}$ «La judicialización antidemocrática de la política», ${ }^{3}$ «La fle-
xibilidad kirchnerista para medir el Lawfare». ${ }^{4}$ Un breve recorrido solo por de la alta presencia que tiene el par política y justicia en el país. Si bien irrumpe con mayor fuerza en algunos períodos, no se trata de ningún modo de un fenómeno nuevo, ni aquí ni en otras partes del mundo. Diversos casos en América Latina o Europa ${ }^{5}$ dan cuenta de esta importancia, tanto política como académica, y que han sido estudiados desde varias perspectivas en los últimos treinta años (Hirschl 2013; Cámara Villar 2000; Martín 2011, 2012; Nosetto 2014, 2017; Sieder, Schjolden y Angell 2016; Belov 2019; Guarnieri y Pederzoli 2002).

Este trabajo forma parte de una investigación mayor ${ }^{6}$ en la que pretendemos estudiar algunos acontecimientos relevantes del par política y justicia durante los Gobiernos de Cristina Fernández de Kirchner (en adelante, CFK), a partir del análisis de un conjunto de piezas discursivas que incluyen distintos tipos y géneros de discurso. Tales acontecimientos (i. e., el envío del proyecto para la reforma del Poder Judicial en el año 2013 con el que trabajaremos en estas páginas, la muerte del fiscal Alberto Nisman en el 2015, ${ }^{7}$ las causas

1 Ver el artículo de Roberto Gargarella, «Por qué el 'lawfare' es un cuento». Clarín, 15 de diciembre del 2020. https://n9.cl/genjx.

2 Ver el artículo de Julio Maier, «Judicialización de la política y política judicial». Página/12, 13 de junio del 2015. https://n9.cl/fp98h.

3 Ver el artículo de Emir Sader, «La judicialización antidemocrática de la política. Página/12, 30 de junio del 2017. https://n9.cl/9uqw2.

4 Ver el artículo de Carlos Pagni, «La flexibilidad kirchnerista para medir el lawfare». La Nación, 19 de mayo del 2021. https://n9.cl/tuejo.

5 Se incluyen aquí denuncias de corrupción; el proceso independentista de Catalunya y el País Vasco; y, más lejano en el tiempo, el proceso conocido como «Mani Pulite» en Italia, etc.

6 Nos referimos a «Operaciones polémicas, liderazgo político y construcción de esfera pública virtual (2011-2019)», financiado por CONICET, así como al PICT 2019 N. 283 , «Operaciones polémicas, liderazgo político y construcción de esfera pública virtual (2015-2019)», financiado por el FONCyT.

7 A comienzo del 2015 apareció muerto el fiscal Alberto Nisman, bajo situaciones que aún se encuentran en investigación. Nisman estaba en clara oposición al Gobierno kirchnerista y llevaba a cabo, hacía algunos años, una investigación en torno al atentado contra la Asociación Mutual Israelita Argentina (AMIA) ocurrido en 1994. Denunció a la expresidenta de pregonar el encubrimiento de iraníes sospechosos de estar vinculados con el atentado 
que tuvieron y tienen a la expresidenta como objeto de denuncia, entre otros) van a ser analizados a partir de recrear una escena ${ }^{8}$ discursiva determinada. Controversia, debate, litigio y polémica serán categorías utilizadas aquí para caracterizar el intercambio - tanto político como periodístico- producido en torno al Proyecto de Democratización de la Justicia presentado por la expresidenta de la nación, Cristina Fernández de Kirchner, en abril del 2013.

En este artículo seguiremos la siguiente estructura: i) una breve contextualización del Proyecto de Democratización de la Justicia; ii) una explicitación de las categorías de análisis y de la metodología del estudio; iii) un apéndice dedicado al análisis de los materiales en torno a lo que entendemos son los tres ejes del debate y que explicaremos próximamente; y, por último, iv) una breve conclusión que abra posibles líneas de reflexión que se desprenden de este escrito.

\section{El kirchnerismo y la reforma judicial}

En Argentina, el kirchnerismo ${ }^{9}$ ha sido reconocido por la implementación de un conjunto de políticas de ampliación de derechos: la Asignación Universal por Hijo, la Ley de Servicios de Comunicación Audiovisual, el Plan Conectar Igualdad, el programa Fútbol para Todos, la aprobación del matrimonio igualitario, la Ley de Identidad de Género, entre otros. Como muchos investigadores trabajaron profusamente (de Diego 2014, Fernández 2016, Becerra y López 2010, Cingolani y Fernández 2019, Dagatti 2017 y 2019; Montero 2012, Montero y Vincent 2013; Malamud y de Luca 2011, Balsa 2013, Vommaro y Aronskind 2010), su vínculo con el sistema mediático y, también, con el Poder Judicial ha sido foco de conflictos. En esta línea, podemos afirmar que la relación entre Gobiernos populares y judicialización de la política no resulta casual: en América Latina, en las últimas dos décadas, la justicia ha sido utilizada como estrategia de disputa contra los Gobiernos, fundamentalmente aquellos vinculados a cierta ampliación de derechos. Sin embargo, tampoco la relación es completamente unívoca:

8 Sobre el concepto de «escena», ver Manguineau (2005) y Montero (2015).

9 Los Gobiernos kirchneristas se desempeñaron en la presidencia durante tres períodos. El primero, a cargo de Néstor Kirchner, del 2003 al 2007. Luego, dos períodos a cargo de Cristina Fernández de Kirchner, del 2007 al 2011 y del 2011 al 2015. Desde el 2019, Cristina Fernández se desempeña como vicepresidenta de la nación integrando la fórmula presidencial junto con Alberto Fernández. Sidicaro (2011) define al kirchnerismo, simultáneamente, como un ciclo de gobierno que comprende tres mandatos y un movimiento político. 
En desmedro de cualquier rápida asociación entre los así llamados populismos y los estados de derecho, los Gobiernos progresistas de la región a los que en general se califica de populistas han desplegado en general su poder de crear instituciones y de instituir derechos como formas legítimas de politización y cambio social. Por tanto, son muchas veces también estos Gobiernos los que alientan la judicialización de ciertas políticas y tienen en los derechos una clave de lectura de su accionar; y muchas otras veces, por el contrario, perciben en la relación entre oposiciones y Poder Judicial una nueva correlación de fuerzas regresiva, con anclaje institucional en el mismo entramado estatal al que supuestamente controlan (Abdo Ferdez 2014, 60)

El puntapié de este vínculo conflictivo entre el kirchnerismo y el Poder Judicial podría situarse en el propio comienzo de la gestión de Gobierno, cuando Néstor Kirchner firmó el Decreto 222/03 y modificó la composición de la Corte Suprema de Justicia. En el marco de una época de desconfianza en las instituciones, la carta ganadora del Gobierno kirchnerista consistió en sentar una posición en contra de la retracción conservadora que se respiraba por entonces en Argentina. Sin embargo, pocos años después, el mismo Kirchner reformó la composición del Consejo de la Magistratura (en adelante, CM), pasando de 20 integrantes a 13 y marcando un sentido sensiblemente opuesto al que pareció avizorarse en el 2003. ${ }^{10}$ Asimismo, y con el fin de comprender el vínculo kirchnerismo-Poder Judicial, resulta necesario destacar que el primero, junto con las organizaciones de derechos humanos y movimientos progresistas, denunciaron la connivencia entre algunos sectores judiciales y el último golpe cívico-militar que tuvo lugar en Argentina en la década de $1970 .{ }^{11}$

Ahora bien, ¿por qué la necesidad de una reforma? Por supuesto que no pretendemos explicar en estas páginas lo que juristas, sociólogos y politólogos continúan preguntándose y analizando, pero sí intentar plantear algunas posibles líneas de respuesta. Algunos investigadores especialistas en derecho, como Bercholc (2013a), proponen tres aristas para intentar responder a la pregunta planteada más arriba: en primer lugar, un componente vinculado a la cuestión coyuntural; en segundo lugar, un punto «de carácter genérico,

10 Un trabajo interesante en este sentido, y escrito al calor de los acontecimientos, es el de Gargarella (2011). Recomendamos su lectura.

11 Un caso ejemplar lo constituye la condena de cuatro exmagistrados de la provincia de Mendoza acusados de no investigar delitos denunciados durante la última dictadura argentina. Ver al respecto https://n9.cl/6hq6n. 
de diseño institucional pero de tipo filosófico-prescriptivo» (Bercholc 2013a, 153); y, por último, un nivel referido al diseño institucional, que responde al cuestionamiento sobre qué, por qué, para qué y cómo.

Detenidos en el primer nivel propuesto por el autor, la propuesta de reforma al Poder Judicial puede explicarse, también, a partir de las dificultades en la promulgación de la Ley de Servicios de Comunicación Audiovisual (LSCA, 2009), ley presentada como un proyecto de «democratización» del acceso a los medios y considerada como «la madre de todas las batallas». ${ }^{12}$ Las disputas entre el Ejecutivo nacional y los principales grupos mediáticos del país concluyeron, las más de las veces, en los pasillos judiciales: de aquí, por lo tanto, una de las vías de solución encontradas por la gestión de Fernández de Kirchner. El caso ejemplar está dado por el Multimedios Clarín quien, a partir de sucesivas medidas cautelares, dejó en una suerte de estado virtual de «suspensión» la aplicación de la nueva ley de medios. Además del punto señalado, que atiende específicamente a las medidas cautelares contra el Estado, Bercholc (2013b) coincide en señalar que la reforma presentada responde a:

la parálisis en la que se encuentra el Consejo de la Magistratura generando una demora inusitada en el proceso de designación de jueces y la consecuencia de un hecho gravísimo, la enorme cantidad de juzgados de distintos fueros que carecen de juez titular y se hallan ocupados por jueces subrogantes y/o interinos; y las demoras, también excesivas, en el avance de los procesos por violación de los derechos humanos en ocasión de la represión del terrorismo de Estado (Bercholc 2013b, 179)

Asimismo, desde el discurso presidencial, se presentó la reforma como una necesidad de terminar con el poder corporativo de jueces y abogados en tanto «los profesionales, por propia definición — las profesiones vienen desde la Edad Media - son corporativos; lo son los médicos, lo son los abogados, lo son los jueces» (CFK, 01-03-2013). ${ }^{13}$

Nos interesa ahora prestar atención a una escena discursiva y polémica en particular. Lo importante en este punto no consiste tanto en la verificación de si lo estudiado se corresponde efectivamente con un proceso de lawfare o, a

12 Ver el artículo «'La madre de todas las batallas es una nueva ley de radiodifusión'». La Nación, 13 de abril del 2008. https://n9.cl/rzdb3.

13 Ver el mensaje a la Asamblea Legislativa de Cristina Fernández de Kirchner, el 1 de marzo del 2013. https://n9.cl/ufp3c. 
la inversa, con un intento de un determinado Gobierno de interceder - con fines político partidarios - en el correcto funcionamiento de la justicia sino, más bien, cómo esos procesos político-judiciales que estudiaremos aparecen así tematizados en la escena pública argentina.

Comenzaremos, tal como adelantamos, por la presentación del Proyecto de Democratización de la Justicia, realizada el 8 de abril del 2013 por la expresidenta Cristina Fernández de Kirchner. El anuncio oficial se realizó en el Museo del Bicentenario cuando Fernández comunicó el envío al Congreso de un proyecto de ley con seis iniciativas para reformar el sistema judicial de Argentina: i) ingreso democrático al Poder Judicial, al Ministerio Público Fiscal y a la Defensa; ii) publicidad de los actos del Poder Judicial; iii) publicidad y acceso a las declaraciones juradas; iv) reforma del Consejo de la Magistratura; v) creación de tres nuevas Cámaras de Casación; y vi) regulación de cautelares contra el Estado. A pesar de la aprobación de tres de las seis iniciativas en la Cámara de Diputados, y de las tres restantes en la Cámara de Senadores, en junio de ese año la Corte Suprema de Justicia de la Nación (en adelante, CSJN) declaró inconstitucionales los artículos 2, 4, 18 y 30 de la Ley 26.855 y el Decreto $577 / 13 .{ }^{14}$ Las primeras tres iniciativas contaban con relativa aprobación dado que proponían darle mayor visibilidad y transparencia a un poder que, en general, se ha caracterizado por cierta opacidad. Las tres restantes, sin embargo, fueron sobre las que, como veremos, se erigió la disputa.

Por las características del momento histórico en el que esta presentación tuvo lugar, el proceso dicotómico que separa kirchnerismo/antikirchnerismo (también, aunque con mayor amplitud, llamado «la grieta») afloró con toda su fuerza, y el arco político y mediático se sumó a la discusión sobre la reforma del Poder Judicial. Para ello, por ejemplo, se realizó, a mediados de abril, el Congreso sobre Democratización de la Justicia - cuyo título fue, de manera sintomática, «La sociedad le habla a la Justicia»-, del que participaron referentes de organizaciones de derechos humanos como Estela de Carlotto u Horacio Verbitsky, ${ }^{15}$ procuradores, jueces y fiscales de distintas jurisdicciones, intelec-

14 Ver el artículo «La Corte declaró inconstitucional cambios en el Consejo de la Magistratura». Centro de Información Judicial, 18 de junio del 2013. https://n9.cl/3e02d.

15 Estela de Carlotto es la presidenta de la Asociación Abuelas de Plaza de Mayo, referente público por la lucha en torno a la verdad, la memoria y la justicia respecto de los delitos de lesa humanidad cometidos durante la última dictadura cívico-militar. Verbitsky es un reconocido intelectual y militante por los derechos humanos que, en ese entonces, se desempeñaba como presidente del Centro de Estudios Legales y Sociales (CELS). 
tuales e investigadores, y funcionarios afines al kirchnerismo. ${ }^{16} \mathrm{El}$ cierre del Congreso estuvo a cargo de Fernández de Kirchner. ${ }^{17}$ Del lado contrario, la oposición organizó un «cacerolazo» a mediados de abril para oponerse a la reforma, movilización de la que participaron diferentes figuras que, luego, solo vieron crecer su capital político: Mauricio Macri es, a estos fines, el primer ejemplo.

\section{Metodología y categorías de análisis}

Nos interesa, entonces, abordar este acontecimiento de presentación de un proyecto de ley a partir de recuperar la discusión que, entre el discurso político y el periodístico, se dio en torno a tres aspectos que detallaremos seguidamente: el tiempo, su constitucionalidad y la división de poderes. Estamos situados en lo que Verón (1987) denominó «entidades del imaginario político» $\mathrm{y}$, desde allí, contrastaremos el modo en que un discurso político (presidencial, en este caso) y un conjunto de discursos periodísticos intervienen en la discusión pública, en el espacio púbico mediatizado. ${ }^{18}$

En cuanto a las categorías de análisis, quizá sea más apropiado hablar de «controversia», tal como Rennes $(2016,262)$ la define: «confrontación discursiva argumentada y polarizada, la controversia es también considerada, en la mayor parte de los trabajos sobre el tema, como una confrontación 'prolongada', 'durable', 'reiterable', 'reciclable' y 'resistente'». La autora explica que la controversia, al igual que la polémica, se caracteriza por una confrontación virulenta de argumentos polarizados que está atada a un determinado objeto. ¿Cuál es la diferencia? Pues que, en el caso de la polémica, «la polarización es de ambas partes y objeto de una argumentación exhaustiva. En la mayoría de los trabajos, describir una controversia, sea esta religiosa, estética, científica, literaria, filosófica, sociotécnica o política, implica hacer referencia a un complejo de argumentos contrapuestos» (Rennes, 2020, 261).

16 Si bien no será trabajado aquí, mencionamos que el Congreso estuvo dividido en cuatro espacios (carpas) temáticos: ingreso democrático y transparencia en la justicia; la agilización de la justicia y las nuevas cámaras de casación; cautelares y defensa del Estado; Consejo de la Magistratura y participación popular.

17 Se puede consultar el cronograma completo de actividades, junto con los y las participantes, en el siguiente enlace: https://n9.cl/ej9kv.

18 Aunque no será respondida aquí, una pregunta interesante sobre la conformación de colectivos — tanto aquellos construidos en el discurso político como en el mediático- es ¿en nombre de quién/es habla/n?, ¿los ciudadanos?, ¿las corporaciones? Sobre este punto, recomendamos con énfasis la lectura de la tesis doctoral de Mariano Fernández, En su lugar y en su nombre. Disputas por la representación en el espacio público mediatizado (2017). 
Volvamos a nuestro punto. En abril se dirimió, entre oficialistas y opositores, entre políticos y periodistas, cuáles serían los ejes de la controversia. Luego de la lectura del corpus de análisis hemos identificado que la discusión sobre el proyecto se erige en torno, fundamentalmente, a tres puntos: i) el momento en el que es planteada la reforma, ii) la discusión sobre su constitucionalidad, y iii) el debate sobre la división de poderes.

Estos ejes podrían corresponder a lo que Plantin(2012) denomina «scripts» o lo que Rennes (2016) nombra como «repertorio/s argumentativo/s». En el primer caso, el autor detalla que «los scripts proveen el stock de argumentos sobre el fondo [...]. El script es la tópica, los conjuntos de topoï sustanciales, ligados a la pregunta o cuestión» (Plantin 2012, 69). Rennes (2016, 141) utiliza la metáfora del repertorio para dar cuenta de «argumentos extraídos de stocks de topoï preexistentes [...]. Este tipo de abordaje apunta precisamente a hacer visibles las formas de estabilidad y de recurrencia en el interior de períodos históricamente determinados, que pueden remitir al largo plazo».

En el primer caso (a), se trató de la consideración del tiempo dado a la discusión del proyecto: el oficialismo estaba apurado en sancionar la reforma, sin previa consulta, incluso con sus propios legisladores, lo que generaba un halo de sospecha («si lo quieren enviar/aprobar rápido es porque esconden algo»). En el segundo caso (b), la discusión se sostuvo sobre el clivaje constitucional/ inconstitucional, fundamentalmente vinculado a la elección/remoción de los miembros del CM y el artículo ligado a las medidas cautelares contra el Estado («quieren modificar el CM para perpetuarse en el poder»). El último punto (c) remite al respeto sobre la división de poderes: para la oposición, el proyecto de ley significó un avasallamiento sobre el Poder Judicial; para el oficialismo, por el contrario, el Poder Judicial era un órgano corporativo que no respetaba las reglas del juego democrático y, por tanto, debía ser modificado.

La noción de escena con la que trabajamos en este artículo recupera cierto aspecto teatral, el desarrollo de una trama en la que los actores intervienen para darse una representación de sí mismos. Verón $(2001,77)$ afirmaba que «puesta en escena es sinónimo de puesta en sentido: no hay entonces producción de sentido sin puesta en escena». Lo que haremos, por lo tanto, es recrear una «escena controversial» o «litigiosa», esto es, dar cuenta de los intercambios producidos entre dos tipos de discursos —el político y el periodístico-. Se crean aquí «espacios discursivos en los que se pone en juego un litigio, una controversia política, litigio mediante el cual, de acuerdo a Rancière (1996), 
se dirimen asuntos que hacen a la definición de la propia comunidad política» (Montero 2015, 37). ${ }^{19}$ Siguiendo a Bourdieu $(1999,69)$, se trata en ambos casos de discursos de «institución», portavoces autorizados que se permiten hablar en nombre de, «en la medida en que su palabra concentra el capital simbólico acumulado por el grupo que le ha otorgado ese mandato y de cuyo poder está investido». Una pregunta de Verón $(1987,14)$ puede servirnos como faro: «¿En qué medida el análisis de los discursos asociados a estructuras institucionales determinadas permite comprender mejor los mecanismos de dichas instituciones, su naturaleza y sus transformaciones?».

Recrear una escena, tal el objetivo de este artículo, nos obliga a identificar cuáles fueron las voces predominantes que conformaron los distintos puntos de vista sobre los que se estructuró el debate.

En cuanto al discurso político, analizaremos el discurso de presentación del proyecto a cargo de CFK. ${ }^{20} \mathrm{Si}$ bien los actores políticos que intervienen en cualquier discusión pública no se reducen a la figura más importante del Ejecutivo, consideramos necesario - en esta primera instancia - recuperar la voz oficial para poder utilizarla como centro sobre el que se van acoplando las demás voces. Además, y como parte de la disputa con el discurso de los medios opositores, la expresidenta ha utilizado los distintos medios a disposición (redes sociales personales, discursos públicos en eventos oficiales, página web personal, etc.) para responder lo publicado en letras de molde. En tanto controversia pública y mediatizada, la identificación de los ejes de debate descritos previamente nos permite componer un diálogo, aquel que se produce entre el discurso presidencial y el periodístico.

En cuanto al discurso de la prensa, y con base en los tres puntos previamente mencionados (el tiempo, el clivaje constitucional-inconstitucional y la preocupación en torno a la división de poderes), hemos seleccionado el día previo, el día de la presentación del proyecto y el día posterior, esto es, del 7 al 9 de abril del 2013. Los diarios seleccionados fueron La Nación, Clarín y Página/12. La selección de los tres diarios podría explicarse por su masividad; sin embargo, no es este el argumento principal por el cual fueron escogidos. En un análisis sobre el periodismo impreso durante el primer kirchnerismo, y con el fin de indagar el modo de consolidación de los periódicos como actores políticos, de Diego (2014) caracteriza estos tres diarios del siguiente modo:

19 El destacado es del original.

20 El discurso se encuentra disponible en el siguiente enlace: https://n9.cl/t4q5o. 
-Un diario que en todo momento se manifestó como crítico y adversario de la propuesta de recuperación política del primer kirchnerismo: La Nación.

-Un diario que, en los años del Gobierno de Cristina Fernández, se convirtió en el opositor acérrimo y objeto de crítica y repudio por parte del kirchnerismo, sus militantes y sus seguidores: Clarín.

-Un periódico que, desde sus inicios, había sido opositor de los Gobiernos democráticos mediante una lectura satírica de la realidad y que desde 2003 se alineaba con la propuesta oficial por considerarla una articuladora de sus propias líneas políticas: Página/12 (de Diego 2014, 65).

Algunas últimas aclaraciones sobre la conformación del corpus. En la discusión sobre el proyecto de reforma, podemos identificar tres grandes momentos: el primero, sobre el que aquí nos detendremos, relativo a la presentación del proyecto. El segundo, que nuclea a la discusión parlamentaria —el día 17 de abril se llevaron a cabo sesiones especiales en las cámaras de Diputados y Senadores, continuó luego el 24 de abril en Diputados y terminó el 8 de mayo en Senadores-. Y el tercero, con el fallo final de la Corte Suprema de Justicia, en el cual se declararon inconstitucionales varios artículos de la reforma propuesta. En este sentido, este artículo se presenta como una primera aproximación a un problema mayor. Sin embargo, esto no le quita importancia al período aquí analizado: lo interesante será, luego, observar el modo en que cada uno de los argumentos sobre los que aquí nos detenemos fueron desarrollándose, modificándose y resignificándose a lo largo del período.

Entonces, el discurso de CFK por un lado y el de la prensa por el otro conformarán los puntos de vista que analizaremos en estas páginas, sin pretensión de exhaustividad, más bien con el deseo de que la recreación y el análisis de esta controversia nos permita conocer un poco más sobre la institución de un determinado acontecimiento político y cómo este abona en la disputa entre democratización y república.

\section{Análisis}

Antes de adentrarnos en el análisis, retomemos la idea de repertorio argumentativo que desarrolla Rennes (2016) en un trabajo que dialoga no solo con las teorías de la argumentación, sino con la sociología de las manifestaciones. 
Allí la autora precisa dos perspectivas en torno a la idea de stock argumentativo y su vínculo con la mirada histórica:

Por un lado, se busca prioritariamente definir estructuras formales de razonamiento y de argumentación transversales a la mayor variedad de corpus y de situaciones de comunicación. Por otro lado, se tiende más bien a dar cuenta de modos de razonamiento y de argumentación propios de los posicionamientos, de las arenas y/o de los géneros discursivos históricamente determinados. En este segundo enfoque, se renuncia a la perspectiva trans- (o a-) histórica para adherirse a la historicidad de las ideologías, de los «discursos» o de las «formaciones discursivas». Pero, a diferencia de la perspectiva abierta por Michel Foucault (1969), una historia discursiva, en cuanto se constituye desde un prisma argumentativo, implica tomar en consideración los fenómenos de reorientación de esos discursos en dispositivos de persuasión, de refutación, de realineamientos, en suma, una focalización en los procesos de conflictividad social (Rennes 2016, 142). ${ }^{21}$

Uno de los modos de ingresar a los materiales que hemos seleccionado para el análisis lo constituye el propio título del proyecto: «democratización de la justicia». Resulta esclarecedor reflexionar sobre este aspecto, en tanto el nombre dado a un acontecimiento tiene la capacidad de delimitarlo, de darle estabilidad. Hablar de democratización es poner el acento en el proceso: no es ya el interés en la democracia sino en la democratización, «como si ahora esa democracia no fuera otra cosa que el proceso de su propia afirmación, de su propia realización, de su propia profundización» (Rinesi 2013, 24). Hay un desplazamiento, entonces, de la idea de democracia como un horizonte, un lugar de llegada, a «la idea de la democratización como el nombre mismo de ese movimiento, y del énfasis en la libertad al énfasis en los derechos» (Rinesi 2013, 25). El título del proyecto es, por cierto, una primera clave de lectura. ¿Quién podría oponerse a algo que se anuncia como democratizador?, ¿en qué sentido se puede discutir una ampliación de derechos — tal el planteo del oficialismo? Smulovitz (2013) ${ }^{22}$ afirma que la democratización de la justicia, en la sociología jurídica, da cuenta de «las condiciones que facilitan o dificultan el uso y acceso a las cortes por parte de los usuarios más débiles»;

21 Las cursivas son nuestras.

22 Ver el artículo de Catalina Smulovitz, «Sobre la reforma judicial». Foco Económico, 5 de mayo del 2013, https://n9.cl/4tjyo. 
esto es tanto por falta de acceso de recursos económicos y/o simbólicos, «o porque los usuarios utilizan el sistema en forma ocasional y en consecuencia son inexpertos o cuentan con horizontes de tiempo menores para negociar soluciones». Declararse opositor a la idea de democratizar la justicia es declararse en contra del acceso de los más débiles. Pero afirmarse a favor o, mejor aún, como responsable de un proceso de democratización — esta es la carta del oficialismo- es también presentarse como el facilitador (o defensor) de semejante derecho.

Si la pregunta es qué significa la democratización de la justicia, qué aspectos se democratizarían, etc., una primera respuesta ya aparece en una nota de opinión publicada en el diario La Nación el día después de la presentación del proyecto. Joaquín Morales Solá, un periodista de renombre del matutino, afirmó:

La «democratización de los medios» terminó con la mayor concentración de medios oficialistas que se recuerde desde los tiempos de Raúl Apold, durante el primer peronismo. Ahora, la «democratización», la «transparencia»y la «legitimidad» de la Justicia (¿quién podría estar en desacuerdo con esas palabras?) esconde el proyecto final del Gobierno de derribar el último límite de su monumental poder. En síntesis: la Justicia será kirchnerista o no será. ${ }^{23}$

Similares apreciaciones aparecen en el diario Clarín que por esos días inauguró un cintillo: «La reforma $\mathrm{K}$ de la justicia». Quedaron allí agrupadas la mayor parte de las noticias o editoriales sobre el tema. Es decir, lo que «para ellos es democratización», «para nosotros es kirchnerización».

Por el otro lado, en el discurso de presentación del proyecto, CFK afirmó que «Democratización de la Justicia no implica que ningún magistrado ni ningún funcionario, en particular, deba sentirse como que estamos tratando de antidemocrático al poder». En un artículo anterior (Gindin 2019), en el que comenzamos a analizar este vínculo discursivo conflictivo entre el discurso de CFK y el lugar que ocupa y se le asigna al Poder Judicial —y a los actores en él involucrados-, decíamos que ese fragmento de discurso sugiere, a partir de una negación metadiscursiva (García Negroni 2009), la anulación de un marco de discurso anterior. «Ese segmento refutativo recoge decires de actores que circulaban en el espacio mediático: tanto las declaraciones de los ma-

23 Ver el artículo de Joaquín Morales Solá, «Derribar al Poder Judicial». La Nación, 9 de abril del 2013. https://n9.cl/qa6ou. 
gistrados opositores como de los sectores políticos de la oposición sostenían aquello que aquí aparece refutado» (Gindin 2021). Esta democratización es, claro, puesta en suspenso en los diarios que analizamos. El principal recurso es el uso de las comillas que, en los diarios opositores, funciona doblemente: como cita y como distanciamiento. Un ejemplo se puede observar en una nota publicada por el diario Clarín el 8 de abril donde, en la bajada, se afirma «La propuesta de Cristina Kirchner de 'democratizar la Justicia' dejó muchas dudas y sospechas», ${ }^{24} \mathrm{O}$ «En algunos sectores, creen que los proyectos de 'democratización' esconderán otro avance del Gobierno sobre el Poder Judicial». ${ }^{25}$ En esta misma línea se pronunciaron algunos sectores de la oposición que publicaron un documento replicado en varios medios $^{26}$ en el que afirmaron que ««Detrás del título meramente retórico de 'democratizar la justicia' se esconde un intento de suprimir la independencia de los jueces, modificando el régimen republicano que establece la Constitución, en perjuicio de las libertades y derechos de todos los ciudadanos argentinos»»». ${ }^{27} \mathrm{Se}$ anticipa aquí, el tercero de los ejes que mencionamos, aquel que busca problematizar la división republicana de poderes.

Por lo tanto, si del lado del oficialismo se afirma que el proyecto busca democratizar la justicia (su acceso, su participación, la transparencia en las decisiones tomadas, la vinculación con los otros poderes del Estado); en el otro lado de la grieta se enfatiza sobre la idea de control: «Con seis proyectos de ley [Cristina] trata de ampliar su control político sobre los tribunales», ${ }^{28}$ o «Los proyectos del Gobierno no buscan 'democratizar' la justicia, 'sino dominar'». ${ }^{29}$ Una sentencia que podría resumir la postura de la oposición en torno a la idea de democratización es la siguiente, publicada por el diario La Nación: «El cristinismo suele usar términos del deber ser para convertirlos luego en lo que no debe ser». ${ }^{30} \mathrm{El}$ periodista se refiere aquí no solo al intento de aprobación de la reforma judicial, sino a otros procesos que el kirchne-

24 Ver el artículo «Las dudas y sospechas que deja la propuesta de Cristina». Clarín, 8 de abril del 2013. https://n9.cl/9ln5d.

25 Ver el artículo «Dudas en la Justicia por los anuncios de Cristina». Clarín, 8 de abril del 2013. https://n9.cl/ dzqd6.

26 Si bien el documento fue publicado con posterioridad a las fechas que aquí se recortaron como corpus de análisis, resulta interesante mencionarlo por la potencia de sus declaraciones.

27 Ver el artículo de Laura Serra, «Duro documento de la oposición en contra de la reforma judicial». $L a$ Nación, 12 de abril del 2013. https://n9.cl/zslze.

28 Ver el artículo «Cristina lanzó su avance más fuerte sobre el Poder Judicial». Clarín, 9 de abril del 2013. https://n9.cl/dz387.

29 Ver el artículo «Presentan hoy la amplia reforma al Poder Judicial». La Nación, 8 de abril del 2013. https:// $\mathrm{n} 9 . \mathrm{cl} / \mathrm{d} 2 \mathrm{~g} 5 \mathrm{t}$.

30 Ver el artículo de Joaquín Morales Solá, op. cit. 
rismo presentó como «democratizadores» tal como la mencionada Ley de Servicios de Comunicación Audiovisual.

\subsection{Los tres ejes del debate}

Como se mencionó arriba, una parte importante de los argumentos en contra del proyecto que fueron publicados por la prensa antikirchnerista $-\mathrm{y}$ que recogían los discursos de los políticos opositores - tenía que ver con el momento en que la reforma fue presentada. Si bien nada indica que dos meses antes o tres meses después el momento hubiese sido considerado como «el indicado» por la oposición — tanto política como mediática—, se consideró que sea por el hermetismo a los puntos sobresalientes del proyecto o por la premura en el envío al Parlamento, el proyecto aparecía envuelto en un halo de desconfianza. Un día antes de su presentación, el diario La Nación afirmaba: «Nadie conoce, no obstante, el detalle de las iniciativas y ese es otro motivo que esgrime la oposición para no asistir. La reserva es tal que ni siquiera fue informada de los textos la tropa propia». ${ }^{31}$ Por su parte, Clarín sostenía que los anuncios concretos «son una perfecta incógnita para la oposición y para buena parte incluso del kirchnerismo». ${ }^{32}$

Una vez dadas a conocer las medidas que conformaban el proyecto, el problema del «momento» se trasladó a la inmediatez con la que el Ejecutivo pretendía la aprobación.

Ahora bien, resulta interesante no solo la distinción de los ejes mencionados sino el modo en que se concatenan, se hilvanan entre sí. Esto es, el supuesto apuro del kirchnerismo en presentar y aprobar el proyecto de ley (primer eje) radica en que parte de las modificaciones son inconstitucionales (segundo eje) y esto es así, en uno de sus aspectos, porque avasalla el Poder Judicial, no respetando la división de poderes (tercer eje).

Como es sabido, las denuncias de corrupción son uno de los pilares en los que se fundan los procesos de lawfare. Esto es, la guerra jurídica implica —o, más bien, necesita de - una tríada que incluye a la oposición, a sectores judiciales y a medios de comunicación. El desafío, entonces, consiste en demostrar —o sugerir, o al menos intentar instalar - que lo que se hace tiene como

31 Ver el artículo de Paz Rodríguez Niell, «Rechazo de los opositores al plan de democratización de la justicia». La Nación, 7 de abril del 2013. https://n9.cl/95qf7.

32 Ver el artículo «Dudas en la Justicia por los anuncios de Cristina». Clarín, 8 de abril del 2013. https://n9.cl/ dzqd6. 
fin ocultar hechos de corrupción. Tal es el ejemplo de la modificación en torno a la elección y la remoción de los integrantes del CM, uno de los artículos que más críticas recibió y que terminó por declararse inconstitucional durante el mes de junio del 2013.

Según la oposición, el cambio propuesto en el CM le permitiría al kirchnerismo la reelección indefinida, pero, sobre todo, tener la venia de los jueces para tratar temas de corrupción del Gobierno. Por el contrario, Irina Hauser, editorialista de Página/12, publicó una nota de opinión ${ }^{33}$ en la que sostuvo que ese cambio, lejos de asociarse con un intento de obtener impunidad, permitiría romper con el poder de las corporaciones de jueces y abogados. Además, en otra nota del diario — que puede ser considerado la «voz oficialista» de la prensa nacional—, la reforma del CM se entiende como «un intento por invertir la ecuación que ha dado una capacidad obstructiva notoria a los radicales en ese organismo». ${ }^{34}$

Ahora bien, si tomamos al discurso presidencial como pivote con el que dialogan los demás discursos, ¿cómo caracteriza CFK a la modificación propuesta?, ¿con qué argumentos justifica su modificación? En primer lugar, es necesario recordar que una de las cuestiones a definir es cuál es el demos con la facultad para elegir la composición del Consejo de la Magistratura. En tanto «organismo de conducción y dirección política» — tal la definición ${ }^{35}$ que CFK le otorga - el CM debe estar sometido a las reglas que rigen al conjunto del poder político. Además, y con el fin de evitar una composición homogénea (con sentido corporativo, según CFK), se impulsó la incorporación de científicos, académicos, contadores, etc., para conformar un consejo interdisciplinario. Votación popular y composición diversa podrían identificarse como los dos puntos sobresalientes que, en el discurso presidencial, justificaron la medida. Como respuesta a ese discurso, el diario Clarín publicó que «en el lado opositor se hace hincapié en un supuesto intento de copamiento por parte del kirchnerismo del Consejo de la Magistratura». ${ }^{36}$ Asimismo, se entiende que la elección popular es inconstitucional porque «la Constitución habla de representantes de

33 Ver el artículo de Irina Hauser, «Las claves de la reforma», Página/12, 9 de abril del 2013. https://n9.cl/ ryxib.

34 Ver el artículo de Irina Hauser, «Tiempos de democratización». Página/12, 7 de abril del 2013. https:// n9.cl/6wlav.

35 Interesante resulta que el diario Clarín lo define como «el organismo que propone y destituye jueces». Al respecto, ver el artículo «Con ataques a la Justicia, Cristina anunció la ampliación de la Magistratura a 19 miembros». Clarín, 8 de abril del 2013. https://n9.cl/ocf5y.

36 Ver el artículo «Dudas en la Justicia por los anuncios de Cristina», op. cit. 
los estamentos, es decir elegidos por sus pares».. ${ }^{37}$ ¿La reforma del Consejo de la Magistratura busca entonces diversificar el acceso al órgano o sirve para que el kirchnerismo tenga más control sobre el Poder Judicial? Adrián Ventura, ${ }^{38}$ en La Nación, advirtió que esta reforma significaba «Menos control judicial sobre el Gobierno. Menos poder para los jueces. Y más política en la justicia».

El proyecto que propuso la regulación de las medidas cautelares contra el Estado fue denunciado también de inconstitucional. Se trataba, en este caso, en lo que la oposición presentaba como una «venganza» contra el Grupo Clarín por la nueva ley de medios y las dificultades en su aplicación. Morales Solá enfatizó esta idea afirmando que dado que al Gobierno le «molestaban» las medidas cautelares debía, por tanto, eliminarlas. En su columna de opinión, ${ }^{39}$ sostuvo que «Las cautelares provocaron enojos en la huésped de Olivos. La limitación a los jueces para decidir cautelares cambiará las cosas: dejaría al Gobierno con un inmenso espacio para perpetrar daños, justos o injustos». La posición exactamente opuesta es la del Gobierno, el cual irónicamente afirmó que dada la cantidad de medidas cautelares contra el Estado presentadas como modo de detener la aplicación de una ley o un decreto, se abría una nueva «rama del derecho, que no existía cuando yo iba a la facultad: el derecho cautelar» (CFK, 08-04-13).

Queda, por último, detenernos rápidamente en el tercer punto a analizar, el relativo a la tematización de la división de poderes. Del lado del oficialismo, tanto en el discurso presidencial cuanto en lo publicado en ese período por Página/12, las razones de la reforma anclan en poder garantizar dicha división y, sobre todo, en resguardar al Poder Judicial de los embates del poder político. La reforma, para la expresidenta, busca entonces otorgarle mayor legitimidad a «uno de los poderes esenciales del Estado». El discurso de CFK en particular y del kirchnerismo en general se ha sostenido en la presentación de sí mismo - y del Ejecutivo que representa — como independiente de las corporaciones, de los monopolios, de los grupos concentrados tanto «de afuera como de adentro». En el marco de un proceso de recomposición de creencia en los dirigentes, los poderes del Estado y los órganos de decisión, el proyecto de democratización se desprende en un curso natural en el que, primero, se recompone el Poder Ejecutivo postcrisis del 2001 y, a partir de allí, se van

37 Ibid.

38 Ver el artículo de Adrián Ventura, «Nueva estrategia del Gobierno para acumular poder». La Nación, 9 de abril del 2013. https://n9.cl/803hd9.

39 Ver el artículo de Joaquín Morales Solá, op. cit. 
recomponiendo los demás órganos, entre los cuales se encuentra, claro está, el Poder Judicial.

En esta escena que hemos reconstruido, los argumentos presentados por la oposición se resumen en una idea harto repetida: «La república está en riesgo». E1 9 de abril el diario Clarín publicó una nota, sin firma, cuyo título «El poder judicial en la mira» adelanta el hilo principal. Verón (2004) afirmaba que el título de las noticias en la prensa gráfica resumía o exhibía el núcleo del tipo de tratamiento que se le iba a otorgar a esa pieza informativa. En este sentido, el encuadre de un determinado acontecimiento tiene como principal función «anticipar ciertas propiedades del discurso que resulta así enmarcado» (Verón 2004, 82). Si algo se tiene en la mira es porque se va a atacar o, al menos, porque se lo observará con detenimiento. Al final de la nota, el diario cita a Gerardo Morales, senador nacional por la provincia de Jujuy, para hacerse eco de sus declaraciones: «Parece que el kirchnerismo no comprende la división de poderes, lo que es tremendo para la democracia que con tanto esfuerzo y dolor recuperó el pueblo argentino hace treinta años. No tienen límites, van por todo». ${ }^{40}$ Ideas similares se publicaron en otra nota del mismo diario, ${ }^{41}$ en la que Francisco de Narváez, senador opositor, sostenía que: «Vamos a resistir el intento de limitar la independencia de la justicia. Están yendo por la Corte». Mario Cimadevilla, senador nacional e integrante del CM, le dijo al diario La Nación: «Cuando las reglas de juego en una república, que están fijadas en la Constitución, a usted no le gustan porque fijan el poder, y usted avanza y las cambia, se puede esperar cualquier cosa». ${ }^{42}$

Democratización y república, pueblo y gente, justicia social y división de poderes. La intervención del Estado y la apuesta por la «democratización de la justicia» era leída por la oposición como un intento «de chavinización de la Argentina» (Vommaro 2017, 16).

\section{Reflexiones finales}

En este trabajo nos hemos encargado de recrear una escena. No cualquier escena, sino una controversial, litigiosa. Y no cualquier litigio sino aquel que tuvo

40 Ver el artículo «El Poder Judicial en la mira», op. cit.

41 Ver el artículo «La reforma $\mathrm{K}$ de la justicia. La oposición denuncia que es un intento de controlar la Justicia». Clarin, 9 de abril del 2013. https://n9.cl/bf0ic.

42 Ver el artículo «Advierten que la reforma de la Justicia pone en riesgo la República». La Nación, 9 de abril del 2013. https://n9.cl/prh2x. 
lugar en el marco de la presentación del «Proyecto de Democratización de la Justicia» en el año 2013 durante el segundo mandato de Cristina Fernández de Kirchner. Para ello, se trabajó con un puñado de notas publicadas en los diarios argentinos La Nación, Clarín y Página/12, y con el discurso de CFK en el que se anunció el envío del proyecto para su discusión parlamentaria. En este marco, distinguimos tres ejes en los que se estructuró el debate: i) el relativo al momento en que se presentó el proyecto y al tiempo dado a la discusión parlamentaria; ii) aquel vinculado a la constitucionalidad o inconstitucionalidad de dos medidas fundamentales, el cambio en la composición del Consejo de la Magistratura y la que se propuso regular las medidas cautelares contra el Estado; y iii) la discusión en torno a la división de poderes, al alcance de la medida en términos de democratización del Poder Judicial. Cualquier recorrido por los medios masivos de comunicación y las redes sociales durante esa época nos devuelve la emergencia de dos gramáticas: la movimentista y la liberal (Natalucci 2018). Presentado como un Gobierno «nacional, popular y democrático», el kirchnerismo atravesó, con amplias consecuencias, una disputa organizada «entre la democratización y la república como principales denominaciones, pero que aparejaban otras como inclusión social, justicia social, pueblo frente a las corporaciones, o bien libertades individuales, división de poderes y corrupción» (Natalucci 2018, 32). ${ }^{43}$

En las discusiones sobre qué es el Poder Judicial y qué función debe cumplir en el juego democrático, pareciera subyacer —idea nada novedosa, por cierto- la necesidad de construir un poder, con todo su tecnicismo, en un poder imparcial y, fundamentalmente, no-político. Y protegerlo del barro de la política. Proteger al Poder Judicial es, ni más ni menos, que proteger a la propia república... Pero ¿qué república?, ¿y protegerla de quién? Es esta construcción la que entra en conflicto entre el oficialismo y la oposición, aun cuando sabemos que el propio espacio judicial se ha convertido en una arena de disputas.

Una interpretación literal de la idea fuerza «democratizar la justicia» debería entenderse como la adaptación de los principios formales democráticos de participación en el proceso de toma de decisiones, de igualdad a efectos de designaciones sin privilegios estamentales y/o procedimentales, $\mathrm{y}$ de aplicación de las reglas de las mayorías a tales fines. También se pueden esbozar diseños «de-

43 Las cursivas son del original. 
mocratizadores» pero más apegados a las ideas «democrático-representativas», por ejemplo, aumentar el número de jueces en los más altos tribunales a fin de lograr una base de funcionarios judiciales que permita una óptima representación sociológica-espejo de los distintos clivajes representables de la sociedad (Bercholc 2013, 180).

Los Gobiernos argentinos han intentado, con mayor o menor éxito, distintas reformas del Poder Judicial, reformas todas que han sido anunciadas como «democratizadoras». La importancia creciente que adquieren los tribunales de justicia en lo que se denomina co mo la judicialización de la política hace que el par política y justicia adquiera cada vez más importancia en la discusión pública: la prensa y el discurso político son, en este aspecto, una muestra de ello. 


\section{Referencias bibliográficas}

Abdo Ferez, C. 2013. La política y la juristocracia. Sociedad, (33), 51-72. https://n9.cl/ n3ztg.

Balsa, J. (Coord.). 2013. Discurso, política y acumulación en el kirchnerismo. Quilmes: Universidad Nacional de Quilmes.

Bercholc, Jorge. 2013a. Notas para entender la reforma judicial que propone la «democratización de la justicia». Cuadernos Manuel Giménez Abad, (5), 158-163.

Bercholc, Jorge. 2013b. Reforma judicial y democratización de la justicia en la Argentina. La compleja articulación entre la democracia y la república. Iberoamericana, XIII(50), 179-184.

Becerra, M. y López, S. 2010. La contienda mediática. Revista de Ciencias Sociales, (16), 9-30. https://n9.cl/f59iz.

Belov, M. 2019. Courts, Politics and Constitutional Law: Judicialization of Politics and Politicization of the Judiciary. Londres: Routledge.

Bourdieu, P. 1999. El lenguaje autorizado: las condiciones sociales de la eficacia del discurso ritual. En ¿Qué significa hablar? Madrid: Akal.

Cámara Villar, G. 2000. Justicia y política en la España democrática (una reflexión a propósito de los llamados «jueces estrella» y la judicialización de la política). Revista de Derecho Político, (47), 27-52, https://n9.cl/o2fht.

Cingolani, G. y Fernández, M. 2019. Cristina, un espectáculo político. Cuerpos, colectivos y relatos en la última presidencia televisiva. Buenos Aires: Editorial Prometeo.

de Diego, J. 2014. La prensa escrita durante el gobierno de Néstor Kirchner. Periodismo de opinión y disputas por el sentido político frente al proceso de construcción del kirchnerismo. Los casos de Clarín, La Nación y Página/12 (tesis doctoral). La Plata: Universidad Nacional de La Plata.

Gindin, I. 2019. Mi aparente fragilidad. Buenos Aires: Prometeo Editorial

Gindin, I. 2021. Cristina Fernández y el Poder Judicial. Apuntes sobre un vínculo -discursivo- conflictivo. Revista Mexicana de Opinión Pública, año 16 (30), 59-73. doi 10.22201/fcpys.24484911e.2021.30.71082

García Negroni, M. 2009. Negación y descalificación: a propósito de la negación metalingüística. Revista Ciências \& Letras, (45), 61-82, https://n9.cl/pdghi.

Dagatti, M. 2017. El partido de la Patria. Los discursos presidenciales de NK. Buenos Aires: Biblos.

Dagatti, M. 2019. La vida por las ideas. Los discursos públicos de Néstor Kirchner (2006-2009). Villa María: Eduvim. 
Fernández, M. 2017. En su lugar y en su nombre. Disputas por la representación en el espacio público mediatizado (tesis doctoral). La Plata: Universidad Nacional de La Plata.

Gargarella, R. 2011. Notas sobre kirchnerismo y justicia. En Malamud, A. y de Luca, M. (Coords.). La política en tiempos de los Kirchner (pp. 63-70). Buenos Aires: Eudeba. Guarnieri, C., y Pederzoli, P. 2002. The Power of Judges: A Comparative Study of Courts and Democracy. Nueva York: Oxford University Press.

Hirschl, R. 2011. The judicialization of politics. En Goodin, R. (Ed.). The Oxford Handbook of Political Sciencie. Nueva York: Oxford University Press.

Maingueneau, D. 2005. ¿ «Situación de enunciación» o «situación de comunicación»? Revista electrónica Discurso.org, 4(7).

Malamud, A. y de Luca, M. (Coords.). 2011. La política en tiempos de los Kirchner. Buenos Aires: Eudeba.

Martín, L. 2012. Las formas del giro judicial. Judicialización de la política en la democracia argentina contemporánea. En Cheresky, I. y Annunziata, R. Sin programa, sin promesa. Liderazgos y procesos electorales en Argentina, compilado (pp. 203-237). Buenos Aires: Editorial Prometeo.

Martín, L. 2011. Giro judicial y legitimidad pública en la política argentina. En Cheresky, I. (Comp.). Ciudadanía y legitimidad democrática en América Latina (pp. 363-393). Buenos Aires: Editorial Prometeo.

Montero, A. S. 2015. Memoria y litigio. Los debates sobre las «Leyes del perdón» en Argentina y Uruguay. Clepsidra, Revista Interdisciplinaria de Estudios Sobre Memoria, 2(4), 34-65. https://n9.cl/auimg.

Montero, A. S. 2012. ¡Y al final un día volvimos! Los usos de la memoria en el discurso kirchnerista (2003-2007). Buenos Aires: Prometeo.

Montero, A. S. y Vincent, L. 2013. Del «peronismo impuro» al «kirchnerismo puro»: la construcción de una identidad política hegemónica durante la presidencia de NK en Argentina (2003-2007). Postdata, 18(4), 123-15. https://n9.cl/0kr2q.

Natalucci, A. 2018. Entre la democratización y la república. Revisitando el ciclo de movilizaciones en el último gobierno kirchnerista (Argentina, 2012-2015). Estudios de Derecho, 75(166), 30-50. https://n9.cl/4acit.

Nosetto, L. 2017. Judicialización de la política y legitimidad democrática. En García Delgado, D. y Gradín, A. (Comps.). El neoliberalismo tardio. Documentos de Trabajo N. ${ }^{\circ}$ 5. Teoría y Praxis (pp. 203-214). Buenos Aires: FLACSO.

Nosetto, L. 2014. Reflexiones teóricas sobre la judicialización de la política en la Argentina. Documentos y Aportes en Administración Pública y Gestión Estatal, 14(23), 93-123. https://n9.cl/0tp10. 
Rennes, J. 2020. La controversia política y sus fronteras. Estudios Sociales, XXX(58), 257-283. https://n9.cl/n8zwg.

Rennes, J. 2016. Las formas de la protesta. Sociología de las movilizaciones y teorías de la argumentación. En Montero, A. S. (Comp.). El análisis del discurso polémico. Disputas, querellas y controversias (pp. 139-161). Buenos Aires: Editorial Prometeo.

Plantin, C. 2012. La argumentación: historia, teorías, perspectivas. Buenos Aires: Biblos.

Rinesi, E. 2013. De la democracia a la democratización: notas para una agenda de discusión filosófico-política sobre los cambios en la argentina actual a tres décadas de 1983. Debates y Combates, 5(3), 22-29. https://n9.cl/fy3zl.

Sidicaro, R. 2011. El partido peronista y los gobiernos kirchneristas. Nueva Sociedad, (234), 74-94. https://n9.cl/p0km9i.

Sieder, R., Schjolden, L. y Angell, A. (Eds.). 2016. The Judicialization of Politics in Latin America. Nueva York: Palgrave Macmillan US. https://n9.cl/vc1pu.

Verón, E. 2004. Fragmentos de un tejido. Barcelona: Gedisa.

Verón, E. 2001. El cuerpo de las imágenes. Buenos Aires: Grupo Editorial Norma.

Verón, E. 1987. La palabra adversativa. En El discurso político. Lenguajes y acontecimientos, AA. VV. (pp. 12-26). Buenos Aires: Editorial Hachette.

Vommaro, G. 2017. La larga marcha de Cambiemos. La construcción silenciosa de un proyecto de poder. Buenos Aires: Siglo XXI Editores.

Vommaro, G. y Aronskind, R. (Comps.). 2010. Campos de batalla. Las rutas, los medios y las plazas en el nuevo conflicto agrario. Buenos Aires: Prometeo Editorial. 